\title{
POUR UNE ANALYSE ÉMOTIONNELLE DES DISCOURS POLITIQUES : L'EXEMPLE DES CAMPAGNES PRÉSIDENTIELLES FRANÇAISES (1981-2012)
}

\author{
Marion Ballet ${ }^{1}$
}

Les sciences politiques contemporaines ont longtemps été réticentes à appréhender les dimensions affectives de leurs objets de recherche. On jugeait les émotions trop subjectives et trop éphémères, donc incompatibles avec l'indispensable scientificité et rationalité du chercheur. Et pourtant, il est difficile de nier que les phénomènes politiques étudiés regorgent de manifestations affectives, plus ou moins intenses et plus ou moins visibles. Que l'on songe par exemple aux manifestations, aux cérémonies, aux débats parlementaires ou encore aux allocutions de nos responsables politiques. Cette contribution se propose de jeter les bases d'une analyse émotionnelle des discours politiques. Elle s'appuiera sur l'exemple des campagnes présidentielles françaises entre 1981 et 2012, périodes particulièrement propices à l'activation d'émotions. L'usage de rhétoriques affectives demeure un passage obligé pour les candidats qui entendent influencer leurs électeurs et les inciter à leur accorder leur suffrage.

1 Marion Ballet est Docteure en sciences politiques de Sciences Po Paris et Chercheuse associée au Centre d'Histoire culturelle des Sociétés Contemporaines de l'Université de Versailles Saint-Quentin-en-Yvelines.

Recherches en communication, $\mathrm{n}^{\circ} 41$ (2014). 
La politique démocratique moderne regorge de manifestations affectives plus ou moins intenses et plus ou moins visibles. Que l'on songe à la confiance dans les représentants élus, à la joie de la victoire électorale ou encore au rejet de la violence politique (Ansart, 1983). Et pourtant, elle a cherché à se construire en limitant au maximum la légitimité des émotions, valorisant l'idée de décideurs et de citoyens rationnels, c'est-à-dire affranchis des passions qui fausseraient leur jugement et altèreraient leurs décisions (Rosanvallon, 1992, pp. 105-129). Cette « doxa démocratique » reprend donc à son compte l'idée cartésienne, aujourd'hui largement invalidée (Damasio, 1995 ; Nussbaum, 2001 ; Braud, 1996), d'une opposition entre la raison, domaine de l'esprit et de la réflexion, et l'émotion, domaine du corps et de l'intempérance.

Ce sont ces mêmes préjugés à l'égard des affects qui expliquent la réticence des sciences sociales contemporaines, et notamment de la sociologie, à investir les dimensions affectives de leurs objets de recherche, qui pourtant foisonnent aussi d'émotions : émeutes, rituels politiques, débats parlementaires sont autant d'exemples de manifestations affectives et d'échanges passionnés. Les affects ont longtemps été vus comme des objets d'étude illégitimes. On les juge subjectifs, donc incompatibles avec la scientificité dont se réclament les sciences sociales et la neutralité exigée du chercheur. "Citoyen sentimental » (Marcus, 2002), le chercheur serait forcément affecté par les émotions qu'il entend étudier et y porterait un jugement nécessairement normatif. Finalement, tout s'est passé comme si «l'effort de rationalisation dans l'interprétation de notre vision du monde avait eu pour corollaire l'éviction ou la relativisation de sa dimension affective et passionnelle » (Gauthier \& Le Cour Grandmaison, 2002, p. 8).

Il faut attendre les années 1980 pour que l'étude des émotions commence réellement à se développer aux Etats-Unis (Neuman et al., 2007). Avec la remise en cause du paradigme du choix rationnel, dans la décennie suivante, s'ouvre un nouvel espace pour les travaux promouvant le rôle des émotions dans le processus de décision politique - que ce soit en psychologie ou en sociologie (Edelman, 1988 ; Marcus et al., 1993 ; Sears, 1993). En France, c'est au milieu des années 1990 qu' apparaissent, puis se développent, des travaux réhabilitant la place de l'émotionnel dans la vie politique (Braud, 1991) et des thèmes de recherches renvoyant au registre affectif : souffrance (Boltanski, 1993 ; Revault d'Allonnes, 2008), colère (Traïni, 2008), surprise (Dabene et al., 2007), etc. 
Peu de travaux, en revanche, analysent concrètement les mécanismes de production et de diffusion des émotions politiques. C'est dans cette optique que le présent article entend exposer une méthode empirique d'analyse des affects dans les discours politiques. Développée dans un écrit antérieur (Ballet, 2012), cette méthodologie sera appliquée au cas particulier des campagnes présidentielles françaises de 1981 à 2012, et aux 657 discours retenus dans le corpus.

\section{Appréhender et analyser les émotions : éléments de méthode}

Comme d'autres disciplines, les sciences du langage ont porté une attention de plus en plus grande aux phénomènes émotionnels. Des recherches récentes se sont ainsi attachées à décrire la manière dont les émotions étaient construites dans les discours et contribuaient pleinement au processus argumentatif(Micheli, 2010). Elles offrent une base solide pour construire une grille d'analyse empirique du corpus permettant de distinguer et de repérer les émotions présentes dans les discours électoraux. Les différentes catégories d'analyse disponibles sont synthétisées dans le Figure 1 ci-dessous.

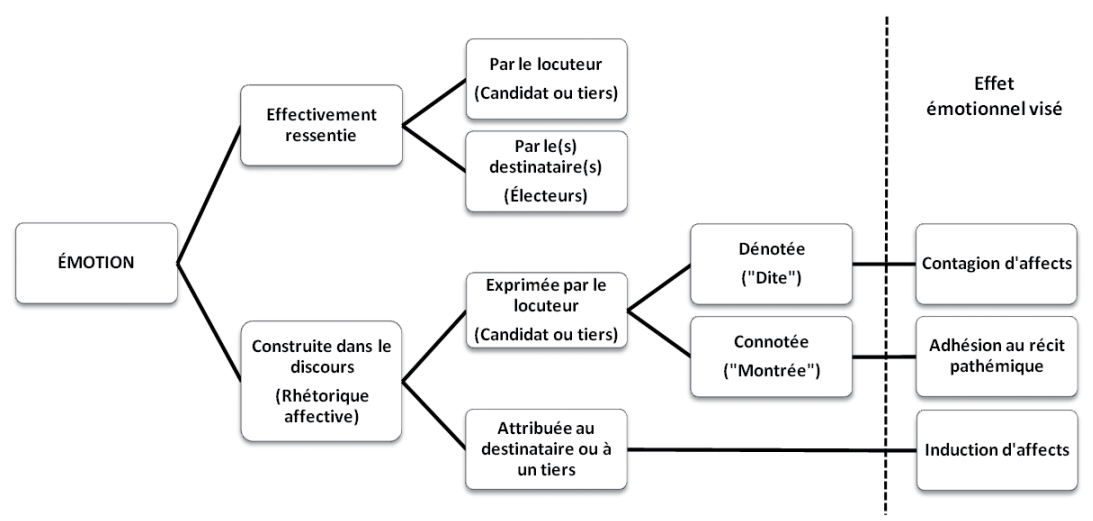

Figure 1 : Catégories d'analyse de l'émotion dans les discours argumentatifs

L'analyse du discours ne permet pas de déterminer ce que ressentent effectivement les participants à une interaction. D'une part, il n'existe aucune preuve d'une correspondance, dans le discours, entre ce qui est exprimé par le locuteur et ce qu'il éprouve réellement (Charaudeau, 2000, p. 135) : la colère peut être feinte, l'optimisme simulé, la compassion non sincère. D'autre part, cette méthode ne fournit 
aucun indice sur l'émotion que ressent effectivement le destinataire du message. L'orateur peut chercher à faire peur ou donner de l'espoir sans y parvenir, voire en produisant l'effet inverse de celui escompté. La méthode proposée ici exclut donc le ressenti effectif du locuteur, impossible à déterminer de manière certaine, et l'effet émotionnel réel que produit le discours sur les destinataires, qui nécessiterait un autre protocole d'enquête.

Il ne sera donc question que des émotions construites dans le discours, que nous nommerons « rhétoriques émotionnelles ». Chacune d'elles se compose de quatre dimensions :

- un stimulus réel ou imaginaire: les émotions s'éprouvent à la vue, l'écoute, ou plus généralement à la représentation d'un objet, d'un événement ou d'une situation (Barbalet, 2002).

- un jugement de valeur : les spécialistes actuels s'accordent sur l'idée que les émotions impliquent un processus d'évaluation des stimuli émotionnels, lui-même conditionné par les schèmes sociaux et culturels véhiculés dans la société (Braud, 1991, p. 101108).

- une visée : par essence, les émotions sont des moteurs à l'action (du latin movere qui signifie " mise en mouvement »). Elles influencent nos comportements et nos jugements, y compris politiques, et leur donnent du sens.

- un registre affectif : peur, indignation, espoir, compassion. Le choix a ici été fait de centrer l'analyse sur quatre registres émotionnels particuliers, à la fois politiquement pertinents (Robin, 2004 ; Ambroise-Rendu \& Delporte, 2008 ; Traïni, 2008) et suffisamment communs pour être partagés par les candidats, par les électeurs auxquels ils s'adressent, mais aussi par le chercheur qui entend les analyser. Chacun de ces registres comporte une gamme d'émotions variées : la peur inclut par exemple la crainte, l'inquiétude, l'anxiété, l'indignation: la colère, le mécontentement, voire dans certains cas la honte (Braud, 2007) ; l'espoir : la confiance, l'optimisme, mais aussi le sentiment d'être rassuré(e), etc. 
Il existe deux grands types de rhétoriques affectives. Les premières reposent sur une «émotion exprimée » (Figure 1) lorsque le locuteur fait directement référence à l'émotion qu'il est censé ressentir. Il la « dit » en la verbalisant par des termes de sentiments ou en la représentant par des gestes ou des expressions faciales culturellement associés à la peur, à l'indignation, à l'espoir ou à la compassion (Calbris, 2003 ; Fonagy, 1983). L'émotion sera dans ce cas « dénotée » (Micheli, 2010) :

Jacques Chirac : "Lorsqu'en qualité de maire de Paris, je me promène dans Paris, je vois les personnes âgées, j'ai toujours peur qu'elles soient agressées par un voyou. » (12 avril 1988).

Jean-Marie Le Pen : « Moi qui suis des vôtres, né du peuple et ma vie durant, resté au service du peuple, quel qu'en soit le prix, c'est confiant et serein que je vous appelle à cette pacifique et décisive bataille. » (20 septembre 2006).

Le locuteur peut aussi " montrer » son émotion, c'est-à-dire l'afficher de manière détournée sans terme de sentiment. Ils élaborent alors des petits récits pathémiques (Charaudeau, 2000) ou émotions « connotées »:

Marie-George Buffet: « Des records de pollution ont été battus en 2005 et le réchauffement climatique est un danger pour l'avenir de la planète. Les pays les plus pauvres sont les plus menacés. » (16 avril 2007).

Jacques-Yves Cousteau : « J'ai été récemment visiter un hôpital à Houston, consacré aux enfants qui sont inguérissables du cancer. On m'avait demandé d'aller les distraire parce que ces pauvres gosses étaient isolés et souffraient énormément. Je suis resté pendant un moment à voir ces gosses de 5 ans, 6 ans, hurlant de douleur, avec leurs mères pleurant à leurs chevets. " (campagne électorale de Brice Lalonde, 14 avril 1981).

La situation d'énonciation revêt une importance essentielle pour pouvoir interpréter les différentes formes d'expression des émotions. Selon les situations, un sourire peut être une marque de joie, d'attendrissement ou de compassion et le terme « hélas » peut exprimer tout autant l'insatisfaction et le regret que la compassion. 
Le second type de rhétoriques affectives se fonde sur l'attribution d'émotions aux différentes instances du discours (Figure 1). Contrairement au cas précédent, ces dernières ne prennent pas ellesmêmes en charge l'énoncé ni n'expriment directement leur émotion. C'est le discours qui leur assigne un état émotionnel, soit en utilisant le vocabulaire pertinent pour dire le sentiment soit en décrivant les traits physiologiques ou comportementaux adéquats :

Nathalie Arthaud: « Sarkozy concentre sur sa personne l'écœurement ou la haine des victimes de la politique menée par la droite depuis cinq ans. » (2012).

Ségolène Royal : « Je vous ai écoutés pour agir juste et pour tenir parole. J'ai entendu vos inquiétudes, vos colères mais aussi vos espoirs. » (2007).

Chaque type de rhétorique affective vise un effet émotionnel particulier : la contagion émotionnelle, l'induction d'affects et l'adhésion au récit pathémique (Figure 1). La première repose sur un partage d'émotions entre le locuteur et ses destinataires. En psychologie, la contagion se définit comme la tendance à imiter les expressions faciales et verbales, les postures et les mouvements d'une autre personne, avec qui l'on converge émotionnellement (Hatfield et al., 1994, p. 5). Contrairement à l'empathie, qui suppose une certaine distance entre le sujet qui l'éprouve et celui qu'il observe, la contagion implique une communion émotionnelle des participants à l'échange. Si elle est plus efficace lorsque les individus sont en présence (c'est le cas par exemple dans une foule ou un cortège), elle peut également être effective lorsque l'échange se fait de manière indirecte (Landowski, 2004, p. 134). Ainsi de la communication politique télévisuelle dont la caractéristique est d'inscrire le spectateur dans son espace-temps et de lui donner une impression de réel. L'orateur peut ainsi exprimer verbalement ou gestuellement sa peur pour chercher à la diffuser dans son public d'électeurs. La rhétorique affective étant toujours soustendue par un jugement de valeur, l'objectif est de légitimer une vision du monde particulière dans laquelle l'objet visant à déclencher la peur (la pollution, l'insécurité, etc.) est effectivement une menace pour les destinataires du message. La contagion sera alors d'autant plus aisée que le locuteur partage avec les auditeurs des similitudes sociales ou idéologiques (Cosnier, 2006, pp. 86-89). 
L'induction d'affects consiste à assigner une émotion à un ou des destinataires (ou plus précisément à l'instance du discours à laquelle sont invités à s'identifier ses destinataires), pour induire chez ces derniers la-dite émotion. L'orateur cherche à faire écho à des prédispositions affectives : la peur pour ses enfants, le sentiment d'être mal considéré, la crainte du nucléaire, l'exaspération contre les fraudeurs, le ressentiment contre les patrons, etc. Il tente aussi de légitimer ces affects particuliers en expliquant les raisons pour lesquelles il convient de les ressentir. L'attribution d'un état psychologique à un sujet se double alors d'énoncés justifiant son bien-fondé (Plantin, 2011, pp. 3-50) :

Charlotte (campagne électorale de Jospin) : « Nous [les femmes] avons d'autant plus de raisons d'être inquiètes que les actions de commandos se multiplient, que les déclarations contre l'IVG et contre la contraception sont de plus en plus nombreuses. » (11 avril 1995).

Par des procédés similaires, le locuteur peut aussi chercher à inhiber ou modérer une propension affective. Dans ce cas, il souligne les raisons pour lesquelles le sujet ne devrait pas éprouver cette émotion :

Cathy Capvert : «Il y a aussi des gens qui s'inquiètent de savoir si la gauche sera présente au second tour. »

Robert Hue : «Mais non. On voit bien maintenant que la droite a fait son choix. Balladur va être éliminé. Il n'y a aucun risque, la gauche sera présente au second tour. » (17 avril 1995).

Les récits pathémiques ont également vocation à activer des prédispositions affectives présentes chez les spectateurs, à la manière des « idiomes rhétoriques » définis par Ibarra et Kitsuse (1993, p. 32). Lorsque les destinataires y adhèrent, ces récits font peur en faisant état d'un danger ; mobilisent l'indignation en évoquant une situation inacceptable, un événement ou un comportement critiquables ; suscitent la compassion en dévoilant la souffrance de malheureux ou donnent de l'espoir en faisant miroiter l'avènement d'une société meilleure. S'accrochant à un objet particulier (ces « pauvres gosses » malades, « les pays les plus pauvres », etc.) et fondés sur un jugement de valeur, ces récits sont d'autant plus efficaces qu'ils font écho aux schèmes de pensée des destinataires à qui ils s'adressent. 
Cette grille de lecture des sources permet de réaliser un premier repérage des différentes rhétoriques affectives de peur, d'espoir, d'indignation et de compassion présentes dans les discours étudiés. Il constitue un préalable à la seconde étape : l'analyse quantitative des rhétoriques émotionnelles. Ce choix de mesurer et quantifier les affects ne va pas de soi, tant la présumée subjectivité et inaccessibilité de ceux-ci semble de prime abord incompatible avec toute quantification. Pourtant, ce type de méthode paraît à la fois pertinent et réalisable. Pertinent car mesurer les rhétoriques émotionnelles permet non seulement de décrire et comparer avec précision les différents usages qui en sont faits par les candidats mais aussi d'éviter l'écueil consistant à se laisser dépasser par la sensation subjective que procurent l'objet et le matériau, la même grille étant appliquée pour l'ensemble des discours, quels qu'ils soient. Réalisable puisque déjà entrepris par des analystes du discours (Rastier, 1995 ; Plantin et al., 2000 ; Micheli, 2010), ce type d'études se centre sur les marques linguistiques de l'émotion, repérables dans les discours et quantifiables au même titre que d'autres constructions linguistiques.

Ce passage à la quantification pose évidemment un certain nombre de difficultés, qu'il convient de dépasser. Le défi principal consiste à passer d'un énoncé parfois complexe à un nombre d'occurrences quantifiées. Il est indispensable de déterminer quoi compter, comment le faire de manière rigoureuse et comment éviter une trop grande perte d'information. La première difficulté consiste à construire une unité de mesure, que nous nommerons occurrence émotionnelle (ou affective), dans laquelle peuvent être converties les deux formes de rhétoriques affectives repérées. En dépit de la distinction opérée entre émotion exprimée et attribuée, celles-ci seront traitées de la même manière. Chaque émotion dite, montrée ou attribuée, que ce soit verbalement ou non, équivaut à une occurrence émotionnelle. Dans l'énoncé suivant, par exemple, ont été dénombrées quatre occurrences affectives (en italique dans le texte) : " [Moue de mécontentement] «Zut! Y'en a assez, voilà. Ça me révolte un peu»» (Waechter, 22 avril 1988). Elles correspondent toutes à des émotions exprimées renvoyant au registre de l'indignation : la première et la dernière sont dénotées (dites), les trois autres connotées (montrées). Ce second exemple - « Depuis 30 ans, les choix politiques ont été mauvais. Pourtant, je veux vous adresser un message d'espoir. » (Le Pen, 2012), comprend deux occurrences affectives (en italique dans le texte). La première correspond à un récit pathémique d'indignation, la seconde à l'expression d'un espoir par le 
biais d'une émotion dénotée. Chaque occurrence émotionnelle a ainsi été repérée puis comptabilisée « manuellement» et une à une.

La seconde difficulté inhérente à la méthode quantitative choisie consiste à catégoriser les stimuli sur lesquels sont construites les rhétoriques émotionnelles, de sorte qu'il soit possible de classer les occurrences affectives non seulement par registre (peur, indignation, espoir ou compassion) mais également par thématique. Les grands thèmes de campagne à partir desquelles se structurent les controverses électorales apparaissent comme des catégories pertinentes, par exemple : l'économie, les questions sociales, les institutions et la vie politique, la sécurité des biens et des personnes, la sécurité extérieure du pays et les Affaires étrangères, les enjeux des droits de l'Homme et des discriminations, 1'Europe, l'environnement, l'immigration, l'éducation et la recherche. Catégories stables d'une élection à l'autre, ces thèmes présentent l'avantage d'exister indépendamment de l'interprétation qu'en font les candidats et de l'importance qu'ils leur accordent dans leur stratégie électorale. Exclusifs les uns des autres (Brugidou, 1995, p. 31), ils permettent d'attribuer à chaque rhétorique affective un seul thème. Ce dernier correspond toujours à l'objet supposé responsable de l'état affectif visé. Dans l'énoncé suivant par exemple : « L'ouverture sur l'Europe mondialiste est un risque mortel pour la liberté, la sécurité et la prospérité des Français " (Le Pen, 13 avril 1995), les deux occurrences de peur (en italique dans le texte) sont rattachées au thème de l'Europe, objet (ou cause) de la menace, et non à ceux de la sécurité ou de l'économie qui correspondent, eux, aux domaines menacés par l'objet dangereux.

$\mathrm{Au}$ terme de cette étape méthodologique, des tableaux de contingence peuvent être établis. Ils recensent et permettent de comparer le nombre d'occurrences émotionnelles par registre affectif et par thème de campagne retenu (par exemple $\mathrm{x}$ occurrences de peur, $\mathrm{y}$ occurrences d'espoir relatives à l'enjeu économique) ; la part (en \%) des occurrences de chaque registre affectif dans l'ensemble des occurrences émotionnelles ( $\mathrm{x} \%$ d'occurrences de peur, $\mathrm{y} \%$ d' indignation, $\mathrm{z} \% \mathrm{~d}$ 'espoir et $\mathrm{v} \%$ de compassion - le tout $(\mathrm{n})$, que nous nommerons « répertoire affectif », correspondant à 100\% des occurrences émotionnelles); la fréquence d'apparition des occurrences émotionnelles dans les supports de communication audiovisuel (1'occurrence toutes les x minutes ou y secondes). 


\section{L'analyse émotionnelle à l'épreuve des campagnes présidentielles françaises (1981-2012)}

Cette méthode d'analyse émotionnelle des discours se révèle être un dispositif de recherche original qui ouvre des pistes de réflexion multiples. Elle constitue d'abord un outil de comparaison intéressant, testé sur un corpus de 657 discours électoraux émis en campagne présidentielle entre 1981 et 2012 (Ballet, 2014). Ceux-ci comprennent 41 déclarations de candidature, 53 professions de foi et 563 émissions de campagne officielle télédiffusées, émises par les candidats de Lutte ouvrière, PCF/Front de Gauche, PS, Verts-EELV, UDF-MoDem, RPR-UMP et Front national, entre 1981 et 2012. Contrairement à d'autres prises de parole plus aléatoires, singulières ou hétéroclites, les documents de propagande sélectionnés demeurent des invariants formels entre 1981 et 2012. Juridiquement régis par des règles de campagne, ils ont aussi l'avantage de placer les candidats sur un pied d'égalité. À ce titre, ils constituent des champs homogènes d'enquête, laissant ouverte la possibilité d'une comparaison dans le temps mais aussi entre candidats.

Cette méthode permet ainsi de faire émerger des évolutions diachroniques qui témoignent des mutations de long terme dans la manière de faire de la politique et de solliciter le suffrage des électeurs. Entre 1981 et 2012, on assiste à une forte hausse du nombre de rhétoriques affectives mobilisées par les candidats à l'élection présidentielle : il passe de 1195 occurrences en 1981 à 1057 en 1988, 1164 en 1995, 1393 en 2002, 1891 en 2007 et 4228 en 2012, soit une hausse sur l'ensemble de la période de $+254 \%$. Cette augmentation est d'autant plus remarquable qu'elle se manifeste dans l'ensemble des supports électoraux étudiés - professions de foi, déclarations de candidatures et clips de campagne (Figure 2). 


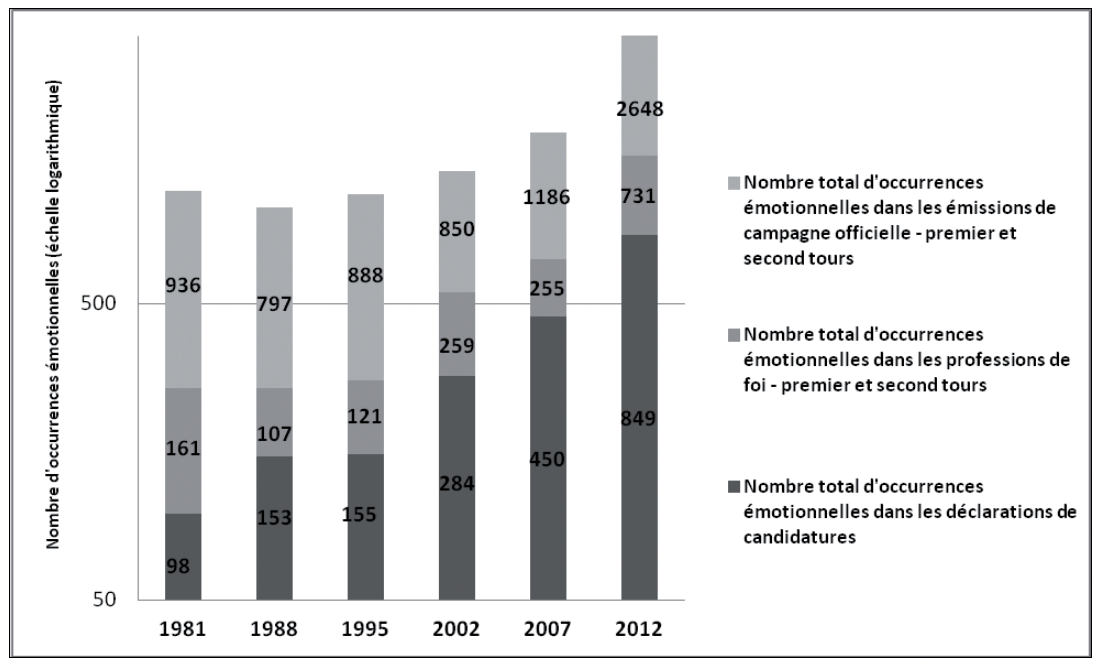

Figure 2 : Evolution du nombre total d'occurrences affectives par document de propagande (1981-2012)

La hausse du nombre d'occurrences affectives dans l'ensemble des discours témoigne d'un dévoilement émotionnel croissant de la part des candidats. Face au développement de l'hyper-individualisme, au déclin des grandes mémoires organisatrices, marxisme en tête (Candau, 1998) ainsi qu'au désintérêt croissant de nombreux Français pour la chose publique, les responsables politiques ont été contraints de se rapprocher des citoyens et de les mobiliser en faisant appel à leur vécu le plus personnel et le plus immédiat. D'un côté, ils se sont mis à exprimer davantage leurs propres affects, témoignage d'un pouvoir politique qui se veut proche et transparent. De l'autre, ils ont pris de plus en plus en charge les émotions des électeurs (leurs souffrances, leurs inquiétudes, leurs aspirations, etc.), tout en cherchant à les activer davantage. Entre 1981 et 2012, le nombre d'occurrences assignant des émotions aux électeurs connaît également une forte hausse (+163\%), passant de 273 en 1981 à 718 en 2012. Cette évolution est particulièrement nette dans les supports audiovisuels. Dans un contexte de professionnalisation croissante des campagnes électorales - marquée par l'essor puis la suprématie du média télévisuel et par le rôle accru des professionnels de la communication -, les candidats ont appris à faire court pour être efficaces. Ils ont intériorisé la nécessité de « faire ressentir l'émotion, [d']aller au nerf » (Saussez, 2004, p. 233) et de 
construire une forme d'intimité avec leur public. Il est à cet égard symptomatique que la réduction de la durée des clips électoraux entre 1981 et 2012 s'accompagne d'une hausse significative, et inversement proportionnelle, de la fréquence d'utilisation des rhétoriques affectives (Figure 3). S'impose finalement l'idée qu'à l'ère de la vidéosphère, la parole politique n'est plus à même, seule, de susciter l'intérêt des électeurs et de garantir la victoire du candidat. Pour être efficace, elle doit impérativement s'associer à la mobilisation émotionnelle qui serait le propre de la communication politique.

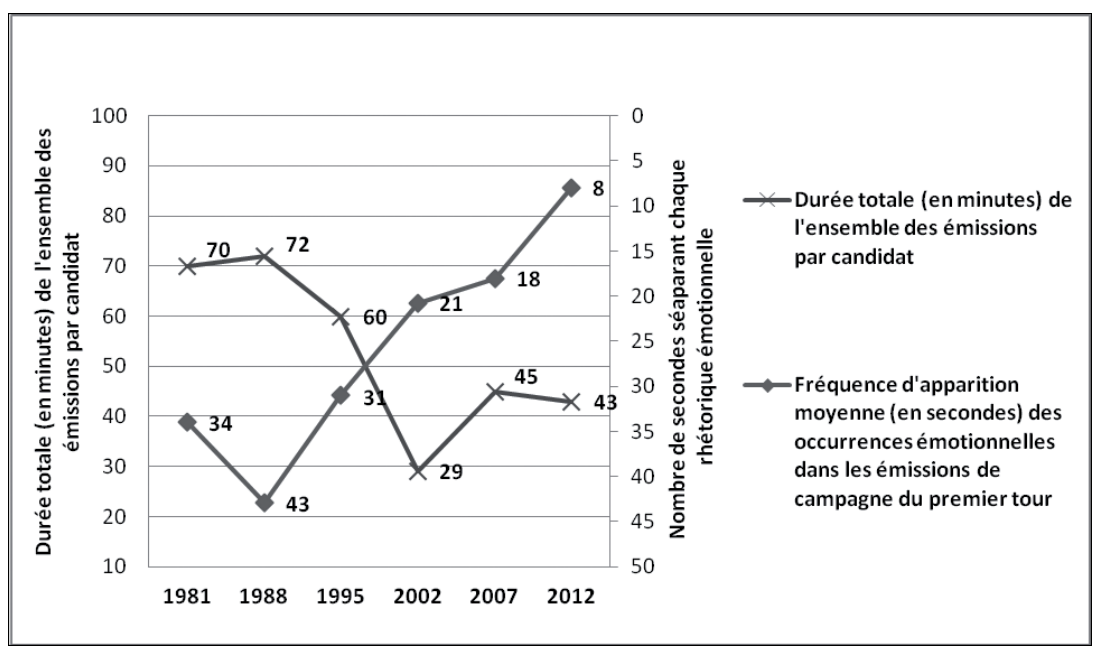

Figure 3 : Évolution comparée de la fréquence d'apparition moyenne (en secondes) des occurrences affectives et de la durée des émissions de campagne officielle au premier tour (1981-2012)

L'analyse émotionnelle des discours électoraux constitue également un outil inédit de comparaison des partis, éclairant sous un jour nouveau la question des identités politiques et des clivages partisans. À la suite d'autres travaux (Inglehart, 1977 ; Braud, 2008 ; Grunberg et al,. 1997 ; Chiche et al., 2000), elle montre ainsi les limites d'une représentation classique de l'espace politique français divisé entre la gauche et la droite. Un clivage prédominant se dessine en effet entre les partis protestataires (hors ou en marge du système) et les partis gestionnaires (dans le système). Les premiers se distinguent d'abord par la forte quantité d'émotions qu'ils mobilisent, toujours plus importante que dans les allocutions des seconds (Tableau 1). 


\begin{tabular}{|c|c|c|c|c|c|c|c|}
\hline \multirow{2}{*}{$\begin{array}{c}\text { PREMIERS TOURS } \\
(\mathbf{1 9 8 1 - 2 0 1 2 )}\end{array}$} & \multicolumn{3}{|c|}{$\begin{array}{c}\text { PARTIS HORS OU EN MARGE } \\
\text { DU SYSTÈME POLITIQUE }\end{array}$} & \multicolumn{3}{c|}{$\begin{array}{c}\text { PARTIS INTÉGRÉS AU } \\
\text { SYSTÈME POLITIQUE }\end{array}$} \\
\cline { 2 - 8 } & LO & $\begin{array}{c}\text { PCF } \\
\text { FG }\end{array}$ & FN & $\begin{array}{c}\text { VERTS } \\
\text { EELV }\end{array}$ & PS & $\begin{array}{c}\text { UDF } \\
\text { MO- } \\
\text { DEM }\end{array}$ & $\begin{array}{c}\text { RPR- } \\
\text { UMP }\end{array}$ \\
\hline $\begin{array}{c}\text { Nb total d'occ. affectives dans } \\
\text { les déclarations de candidature }\end{array}$ & 329 & 557 & 490 & 95 & 131 & 209 & 171 \\
\hline $\begin{array}{c}\text { Nb total d'occ. affectives dans } \\
\text { les professions de foi }\end{array}$ & 416 & 221 & 186 & 199 & 113 & 126 & 119 \\
\hline $\begin{array}{c}\text { Nb total d'occ. affectives dans } \\
\text { les émissions officielles }\end{array}$ & 1266 & 974 & 1048 & 1029 & 567 & 493 & 691 \\
\hline TOTAL (tous supports) & 2011 & 1752 & 1724 & 1323 & 811 & 828 & 981 \\
\hline
\end{tabular}

Tableau 1 : Évolution comparée du nombre total d'occurrences affectives présentes dans les discours des partis hors et dans le système politique au premier tour (1981-2012)

Les partis protestataires, dotés de doctrines et de fonctionnements rigides, trouvent dans les émotions un levier efficace d'identification idéologique et un puissant moteur à l'action. Le second point de clivage tient aux types de registres affectifs privilégiés. Tandis que les discours des partis protestataires sont très fortement empreints d'indignation et très peu d'espoir, ceux des partis gestionnaires véhiculent au contraire beaucoup plus d'espoir et moins d'indignation (Figures 4 et 5).

Répertoire émotionnel moyen des partis hors ou à la marge du système politique $(n=6810)$ Premier tour - toutes élections (1981-20012

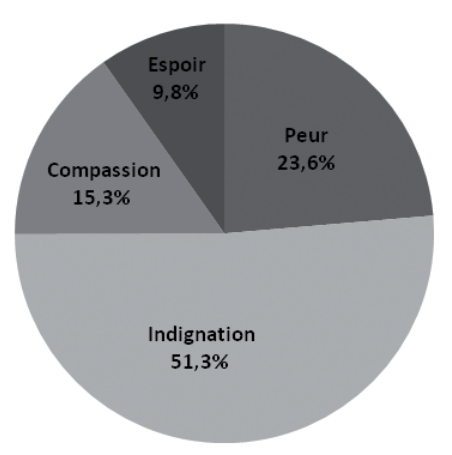

\section{Répertoire émotionnel moyen des partis} gestionnaires $(n=2714)$

Premier tour - toutes élections (1981-2012)

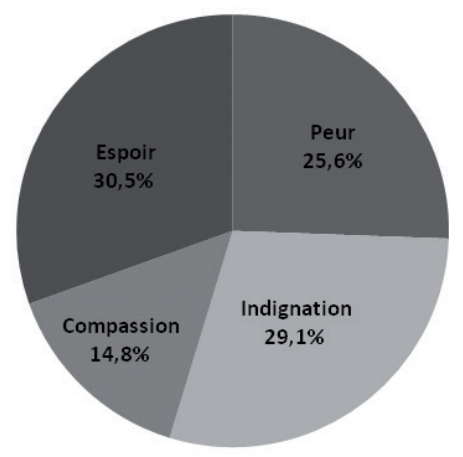

Figures 4 et 5 : Part comparée (en \%) des différents registres affectifs dans les discours des partis protestataires et des partis gestionnaires au premier tour (1981-2012) 
Nés d'un refus de l'ordre établi, les premiers se donnent en effet un objectif de long terme - transformer en profondeur le système politique vis-à-vis duquel ils s'inscrivent en faux -, qui les incite à privilégier le recours aux rhétoriques d'indignation (51,3\% des occurrences affectives recensées dans leurs discours). La part du registre de l'espoir, toujours très peu intense (en moyenne 9,8\%), est à la hauteur des faibles attentes placées dans le scrutin présidentiel. Dans l'impossibilité d'accéder à la présidence de la République (et même le plus souvent au second tour), ces responsables politiques privilégient un futur très éloigné (début d'une nouvelle ère ou d'une renaissance) où pourra être mise en place la société alternative qu'ils appellent de leurs vœux. Rien de tel chez les partis gestionnaires qui ont vocation, au gré des aléas de l'alternance démocratique, à constituer une majorité ou à entrer dans une coalition gouvernementale. Les chances de victoires et l'insertion dans le jeu politique de la $\mathrm{V}^{\mathrm{e}}$ République tendent ainsi à favoriser une forte mobilisation de l'espoir (30,5\% en moyenne) et une plus faible sollicitation de l'indignation (29,1\%).

Au-delà de ce clivage prépondérant, chaque formation politique conserve des spécificités affectives qui lui sont propres. En mettant à jour ces particularités, l'analyse émotionnelle appréhende les mouvements politiques dans le cadre plus général d'une " économie émotionnelle » (Lefranc \& Sommier, 2009, p. 278) qui fonde leur identité politique. Les répertoires affectifs de l'extrême gauche trotskiste et communiste se distinguent ainsi par la prédominance de l'indignation $(63,4 \%$ pour Lutte ouvrière ; $48,3 \%$ pour le PCF et le Front de Gauche) qui vise, sur le terrain économique et social, à mobiliser l'énergie des travailleurs vers l'objectif révolutionnaire. Chez les écologistes, la prééminence des peurs $(40,4 \%)$, en particulier d'ordre environnemental, reflète non seulement la préoccupation originelle du mouvement mais cherche aussi à faire prendre conscience aux citoyens de la nécessité d'agir en urgence pour éviter la perte du genre humain. Les discours d'extrême droite ont eu pour caractéristique de mobiliser fortement la crainte et le rejet de l'Autre. Le registre de la peur $(27,3 \%)$ s'attache d'abord à l'immigration, celui de l'indignation (50,7\%) vise l'Union Européenne, principaux ennemis endogène et exogène de la culture frontiste. Côté partis gestionnaires, le RPR (devenu UMP) se distingue par une posture très optimiste (l'espoir représente $31,9 \%$ de leur répertoire, soit le niveau le plus haut pour l'ensemble des formations étudiées), reflet de leur position privilégiée au sein de la Ve République, régime fondé par l'un des leurs le général de Gaulle. Quant au Parti socialiste, il se 
distingue de ses concurrents de droite par une plus forte mobilisation de l'indignation $(37,9 \%)$ et de ceux de gauche par de plus nombreux appels à l'espoir $(27,4 \%)$.

Cette notion de répertoire émotionnel permet également de repenser la question des leviers de mobilisation des acteurs. À la manière des « répertoires d'action » théorisés par Charles Tilly (1984, 1986), les répertoires affectifs regroupent l'ensemble des ressources émotionnelles à disposition des responsables politiques pour influencer et mobiliser les électeurs. Selon la conjoncture et selon les logiques de positions des acteurs, certaines ressources affectives apparaissent plus opérantes que d'autres. Ainsi par exemple de l'espoir, qui demeure une ressource privilégiée par les candidats sortants, comptables d'un bilan et contraints de prouver que les politiques mises en œuvre alors qu'ils étaient au pouvoir (à l'Elysée, à Matignon ou dans un ministère) sont non seulement satisfaisantes mais aussi porteuses d'espérance pour l'avenir. Ce registre affectif est ainsi toujours plus important dans les répertoires des responsables politiques sortants $(29 \%$ des occurrences émotionnelles présentes dans leurs discours - Tableau 2) que dans ceux des candidats non sortants (12,8\%). Même lorsque les candidats dans l'opposition appartiennent à des partis de gouvernement, la sollicitation de l'espoir demeure supérieure chez les sortants (29\% des occurrences affectives contre $27,5 \%$ pour leurs homologues dans l'opposition).

\begin{tabular}{|c|c|c|c|c|c|}
\hline \multirow{2}{*}{\multicolumn{2}{|c|}{$\begin{array}{l}\text { PREMIER TOUR, MOYENNE DE } \\
\text { TOUTES LES ÉLECTIONS } \\
(1981-2012)\end{array}$}} & \multirow{3}{*}{$\begin{array}{c}\text { SORTANT } \\
497\end{array}$} & \multicolumn{2}{|c|}{ NON SORTANT. } & \multirow{3}{*}{$\begin{array}{c}\text { MOYENNE } \\
1494\end{array}$} \\
\hline & & & TOTAL & $\begin{array}{l}\text { DONT PARTIS } \\
\text { DE GOUV. }\end{array}$ & \\
\hline \multirow{2}{*}{ Espoir } & $\mathrm{Nb}$ d'occ. affectives & & 997 & 366 & \\
\hline & $\begin{array}{l}\text { Part (en \%) de l'ensemble } \\
\text { des occ. affectives }\end{array}$ & 29,0 & 12,8 & 27,5 & 15,7 \\
\hline \multirow{2}{*}{$\begin{array}{c}\text { Ens. des } \\
\text { occ. affectives }\end{array}$} & $\mathrm{Nb}$ d'occ. affectives & 1714 & 7810 & 1330 & 9524 \\
\hline & $\begin{array}{l}\text { Part (en \%) de l'ensemble } \\
\text { des occ. affectives }\end{array}$ & 100,0 & 100,0 & 100,0 & 100,0 \\
\hline
\end{tabular}

Tableau 2 : Part moyenne (en \%) du registre de l'espoir dans l'ensemble des occurrences émotionnelles des candidats sortants et non sortants au premier tour (1981-2012) 
De la même manière, la peur apparaît comme une ressource particulièrement prisée par les chefs de l'État en exercice (Valéry Giscard d'Estaing en 1981, François Mitterrand en 1988, Jacques Chirac en 2002 et Nicolas Sarkozy en 2012). Elle représente 40,7\% des occurrences affectives présentes dans leurs discours, soit un niveau très supérieur à la moyenne de l'ensemble des candidats (26,4\% - Figures 6 et 7).

\section{Répertorie émotionnel moyen des présidents de la République sortants - premier tour} $(1981,1988,2002,2012)(n=1065)$

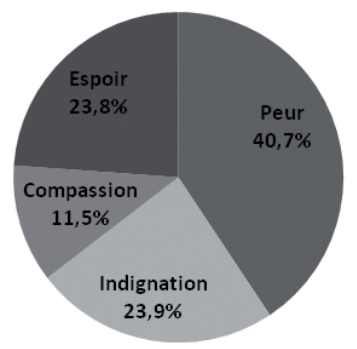

Répertoire émotionnel moyen de l'ensemble des candidats - premier tour $(1981,1988$, $2002,2012)(n=7873)$

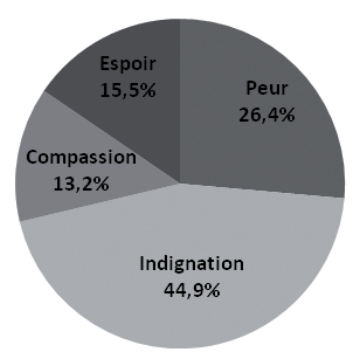

Figures 6 et 7 : Répertoire émotionnel moyen des présidents de la République sortants comparé à la moyenne de l'ensemble des candidats (1981, 1988, 2002, 2012)

Quelle que soit l'élection considérée, la part de la peur dans les répertoires émotionnels des Présidents demeure toujours supérieure à la part moyenne de ce registre pour l'ensemble des candidats. Mieux, sur l'ensemble des campagnes de François Mitterrand, de Jacques Chirac (exception faite de 1981) et de Nicolas Sarkozy, le registre de la peur est le plus intense lorsqu'ils se présentent en tant que Président sortant.

Prenant modèle sur la stratégie payante du premier président de la $\mathrm{V}^{\mathrm{e}}$ République, ces candidats tentent de brandir la menace du chaos afin de se faire réélire (De Gaulle, 1965). Le choix de la peur peut apparaître d'autant plus payant que ce registre émotionnel tend à rassembler les individus derrière une autorité déjà en place. Face aux multiples tensions qui secouent le pays, il reviendrait au Président sortant de maintenir l'intégrité du territoire et de protéger les Français. D'un côté, tous amplifient les menaces qui pesaient sur la France pendant leur Présidence afin de valoriser leur aptitude à remplir la fonction protectrice que le peuple leur a confiée sept ou cinq ans auparavant ; de l'autre, ils dévoilent les risques encourus par le pays s'ils n'étaient pas réélus. 
Nicolas Sarkozy : "Pendant cinq ans, alors que le monde connaissait une succession de crises aussi violentes qu'inattendues, j'ai conduit notre pays. (...) Tout au long de ces crises, j'ai protégé la France et les Français. » (2012).

François Mitterrand : «Tout ce que j'ai vu, je viens de vous le dire, mérite la plus extrême attention. (...) il m'est apparu, et pas à moi seul, que j'étais en mesure de faire front pour éviter ces germes de divisions dont je viens de parler. » (22 mars 1988).

Dernier exemple : la compassion qui a constitué une ressource majeure pour les femmes candidates dans les années 2000. En proie à une crise de légitimité manifeste, la classe politique entend alors " réenchanter » le métier de représentant. Le développement, à cette époque, des stratégies de proximité tend ainsi à valoriser des qualités empathiques supposées féminines : la sensibilité, le dévouement, la capacité à écouter, la douceur, etc. Un plus grand nombre de femmes est appelé à exercer de hautes fonctions afin de mobiliser ces vertus et de « faire de la politique autrement» (Le Bart, 2009, p. 39). Dès lors, le genre peut se transformer en instrument tactique et l'expression compassionnelle devenir une arme, un " capital corporel identitaire » (Achin et al., 2008) pour les candidates qui entendent apporter une réponse « féminine » aux nouvelles précarités sociales. C'est le cas tout particulièrement de Ségolène Royal en 2007 (la compassion représente, en moyenne des deux tours, $23 \%$ de son répertoire affectif). L'espace politique, pour cette dernière, s'ouvre d'autant plus que Nicolas Sarkozy choisit, dans un premier temps, de mettre en avant une virilité populaire, dominante et décomplexée (Achin \& Dorlin, 2008). Dans un tel contexte, valoriser sa féminité permet non seulement de stigmatiser l'agressivité du candidat UMP mais aussi de proposer une solution alternative crédible, fondée sur la tolérance, le respect des individus, voire la douceur. L'effet de ces rhétoriques compassionnelles est d'autant plus fort qu'elles font écho aux préjugés concernant les femmes et les hommes. Face à l'arrogance, à la surdité et à l'agressivité supposés des hommes politiques traditionnels, la candidate valorise son empathie, sa délicatesse, voire sa capacité à supporter le poids de la misère des autres. Finalement, selon la conjoncture électorale, les contraintes et les spécificités de chaque candidat (positionnement politique, rôle endossé, personnalité, genre, etc.), l'usage de tel ou tel registre émotionnel peut 
s'avérer être une ressource essentielle dans la mobilisation des électeurs et même constituer, sous certaines conditions, un « coup gagnant ».

Ce type d'analyse émotionnelle, qui mériterait d'être éprouvée sur d'autres types de corpus et de terrains d'enquête, laisse donc entrevoir de riches pistes de réflexion. D'un point de vue méthodologique, elle offre une grille de lecture des sources à la fois simple et performante qui pourrait tout à fait être étendue à d'autres registres affectifs pertinents : la surprise, la joie, la tristesse, le dégoût, la nostalgie et bien d'autres encore. Les types de comparaison peuvent également être diversifiés : au long court ou sur une période plus restreinte (par exemple les quelques mois d'une campagne, les quelques heures d'un meeting, les quelques minutes d'une émission), entre types de discours différents (oral ou écrit), situations d'énonciation ou orateurs variés (réunion publique, émission de télévision, ouvrage littéraire, interview, débat, etc.). D'un point de vue empirique, l'émotion politique peut être abordée comme un objet d'étude à part entière, dont il convient de comprendre les usages et les fluctuations. Elle peut aussi être utilisée comme un outil pour approcher des phénomènes politiques plus généraux : les identités politiques et les clivages partisans, les alliances électorales et les transfuges entre partis, la volatilité des électeurs et l'engagement des militants. En d'autres termes, l'émotionnel dit tout autant du politique que le politique dit de l'émotionnel.

\section{Références}

Achin, C., \& Dorlin, E. (2008). Nicolas Sarkozy ou la masculine mascarade du Président. Raisons politiques, 31, 19-45.

Achin, C., Dorlin, E., \& Rennes, J. (2008). Capital corporel identitaire et institution présidentielle : réflexions sur les processus d'incarnation des rôles politiques. Raisons politiques, 31, 5-17.

Ambroise-Rendu, A., \& Delporte, C. (Éd.). (2008). L'indignation. Histoire d'une émotion politique et morale, XIXe-XXe siècles. Paris : Nouveau Monde Éditions.

Ansart, P. (1983). La gestion des passions politiques. Lausanne : L'Âge d'Homme.

Ballet, M. (2012). Peur, Espoir, Compassion, Indignation. L'appel aux émotions dans les campagnes présidentielles (1981-2007). Paris : Dalloz.

Ballet, M. (2014). Emotions et élections - Les campagnes présidentielles françaises (1981-2012). Paris : INA Editions.

Barbalet, J. M. (Ed.). (2002). Emotions and Sociology. Oxford : Blackwell.

Boltanski, L. (1993). La souffrance à distance. Morale humanitaire, médias et politique. Paris : Gallimard.

Bongrand, M. (1986). Le marketing politique. Paris : PUF.

Braud, P. (1991). Le jardin des délices démocratique : pour une lecture psycho-affective 
des régimes pluralistes. Paris: Presses de la FNSP.

Braud, P. (1996). L'émotion en politique. Problèmes d'analyse. Paris : Presses de Sciences Po.

Braud, P. (2007). Petit traité des émotions, sentiments et passions politiques. Paris : Armand Colin.

Braud, P. (2008). Sociologie politique. Paris : LGDJ.

Brugidou, M. (1995). L'élection présidentielle : discours et enjeux politiques. Une analyse comparée. Paris : L'Harmattan.

Calbris, G. (2003). L'expression gestuelle de la pensée d'un homme politique. Paris : CNRS Éditions.

Candau, J. (1998). Mémoire et identité. Paris : PUF.

Charaudeau, P. (2000). Une problématisation discursive de l'émotion. À propos des effets de pathémisation à la télévision. Dans C. Plantin, M. Doury, \& V. Traverso (Éd.), Les émotions dans les interactions (p. 135). Lyon : PUL.

Chiche, J., Le Roux, B., Perrineau, P., \& Rouanet, H. (2000). L'espace politique des électeurs français à la fin des années 1990 : nouveaux et anciens clivages, hétérogénéité des électorats. Revue française de science politique, 50(3), 463-487.

Cosnier, J. (2006). La psychologie des émotions et des sentiments. Paris : Retz.

Dabene, O., Hastings, M., \& Massal, J. (Éd.). (2007). La surprise électorale : paradoxes du suffrage universel (Colombie, Équateur, France, Maroc, Suède, Turquie...). Aixen-Provence : Karthala.

Damasio, A. (1995). L'erreur de Descartes : la raison des émotions. Paris : Odile Jacob.

Edelman, M. (1988). Constructing the Political Spectacle. Chicago : University Of Chicago Press.

Fonagy, I. (1983). La vive voix. Paris : Payot.

Gauthier, C., \& Le Cour Grandmaison, O. (Éd.). (2002). Passions et sciences humaines. Paris : PUF.

Grunberg, G., \& Schweisguth, E. (1997). Recompositions idéologiques. Dans D. Boy \& N. Mayer (Éd.), L'électeur a ses raisons (pp. 139-218). Paris : Presses de Sciences Po.

Hatfield, E., Cacioppo, J. T., \& Rapson, R. L. (1994). Emotional Contagion. New York : Cambridge University Press.

Hochschild, A. (1975). The Sociology of Feeling and Emotion: Selected Possibilities. Sociological Inquiry, 45(2-3), 280-307.

Ibarra, P., \& Kitsuse, J. (1993). Vernacular Constituents of Moral Discourse: An Interactionist Proposal for the Study of Social Problems. Dans G. Miller \& J. Hosltein (Éd.), Constructionist Controversies (p. 32). New York : Aldine de Gruyter.

Inglehart, R. (1977). The Silent Revolution: Changing Values and Political Styles among Western Publics. Princeton : Princeton University Press.

Landowski, E. (2004). Passions sans nom. Paris : PUF.

Le Bart, C. (2009). Les présidentiables de 2007 entre proximité et surplomb. Mots. Les langages du politique, 89, 39.

Marcus, G. E. (2002). The Sentimental Citizen: Emotion in Democratic Politics. Pennsylvania : Pennsylvania State University Press.

Marcus, G. E., \& Mackuen, M. B. (1993). Anxiety, Enthusiasm, and the Vote: the Emotional Underpinnings of Learning and Involvement During Presidential Campaings. The American Political Science Review, 87(3), 672-685. 
Micheli, R. (2010). L'émotion argumentée : l'abolition de la peine de mort dans le débat parlementaire français. Paris : Cerf.

Neuman, R. W., Marcus, G. E., Crigler A. N., \& MacKuen M. (Éd.). (2007). The Affect Effect: Dynamics of Emotion in Political Thinking and Behavior. Chicago : University of Chicago Press.

Nussbaum, M. (2001). Upheavals of Thought: the Intelligence of Emotions. New York: Cambridge University Press.

Plantin, C. (2011). Les Bonnes raisons des émotions. Principes et méthode pour l'analyse de la parole émotionnée. Berne : Peter Lang.

Rastier, F. (1995). L'analyse thématique des données textuelles. L'exemple des sentiments. Paris : Didier érudition.

Revault d'Allonnes, M. (2008). L'homme compassionnel. Paris : Seuil.

Robin, C. (2004). Fear: the History of a Political Idea. Oxford : Oxford University Press.

Rosanvallon, P. (1992). Le sacre du citoyen. Histoire du suffrage universel en France. Paris : Gallimard.

Saussez, T. (2004). Le style réinvente la politique. Paris : Presses de la Renaissance.

Sears, D. O. (1993). Symbolic Politics: A Socio-psychological Theory. Dans S. Iyengar \& W. McGuire (Éd.), Explorations in Political Psychology (pp. 113-149). Durham and London : Duke University Press.

Tilly, C. (1984). Les origines du répertoire d'action collective en France et en GrandeBretagne. Vingtième siècle. Revue d'histoire, 4, 89-108.

Tilly, C. (1986). La France conteste de 1600 à nos jours. Paris : Fayard.

Traïni, C. (2008). La musique en colère. Paris : Presses de Sciences Po.

Traïni, C. (Éd.). (2008). Émotions... Mobilisations ! Paris : Presses de Sciences Po.

(2014). Affects, émotions, convictions: l'intime et l'historien. Cahiers de l'équipe de recherche ARCHE, Arts, Civilisation et Histoire de l'Europe, (5).

(2014). Journée d'étude de l'atelier TEXTO sur la mesure des émotions dans les corpus textuels, 12-13 mai 2014, Maison de la Recherche de l’Université Toulouse 2. 